\title{
Active surveillance for highly resistant microorganisms in patients with prolonged hospitalization
}

\author{
Guido J. H. Bastiaens ${ }^{1,2^{*}}$ D, Tom Baarslag ${ }^{3}$, Corinne Pelgrum ${ }^{3}$ and Ellen M. Mascini ${ }^{1,3}$
}

\begin{abstract}
We evaluated a new hospital policy comprising active surveillance for highly resistant microorganisms (HRMO) in patients with prolonged hospitalization, including detection of nosocomial transmission after identification of HRMO carriers. Our findings raise the question of whether active surveillance should be extended from traditional risk groups to patients with prolonged hospitalization.
\end{abstract}

Keywords: Infection control, Surveillance, Nosocomial, Antimicrobial resistance, Cross infection

\section{Introduction}

The rising threat of antimicrobial resistance has been recognized worldwide. In the Netherlands, rates of highly resistant microorganisms (HRMO) have traditionally been low [1]. However, even here, antimicrobial resistance is emerging and the Dutch Ministry of Health, Welfare and Sport regards this matter as having the utmost priority [2].

Besides antimicrobial stewardship, hand hygiene, and transmission-based precautions, active surveillance is important to prevent cross-contamination, allowing detection of patients colonized with HRMO, including methicillin-resistant Staphylococcus aureus (MRSA), vancomycin-resistant Enterococcus faecium (VRE), and carbapenemase-producing Enterobacteriales (CPE). Active surveillance for HRMO in patients at risk due to prior admission to foreign hospitals, residence in refugee and migrant centres or exposure to occupational livestock has been endorsed by Dutch guidelines for years $[3,4]$. Nevertheless, we are often confronted with patients without risk factors, who are unexpectedly tested HRMO-positive, sometimes even as part of an outbreak [1].

\footnotetext{
* Correspondence: GBastiaens@rijnstate.nl

${ }^{1}$ Laboratory for Medical Microbiology and Medical Immunology, Rijnstate Hospital, Pres. Kennedylaan 100, 6883 AZ Velp, The Netherlands

2Department of Medical Microbiology \& Radboud Center for Infectious

Diseases, Radboud University Medical Center, Nijmegen, the Netherlands Full list of author information is available at the end of the article
}

Among other factors, length of in-hospital stay has been positively associated with HRMO-carriage $[5,6]$. To identify HRMO-carriage in patients with prolonged hospitalization a new policy comprising active surveillance for HRMO in patients who have been hospitalized $\geq 14$ days has been endorsed. By isolating newly identified, hospitalized HRMO-carriers and by performing source and contact tracing investigations, the new policy aims to reduce transmission of previously unnoticed HRMO in our hospital. We assessed the impact of this new policy.

\section{Methods \\ Hospital setting and study design \\ This retrospective, nonrandomized observational study was conducted at Rijnstate, an 809-bed, teaching hos- pital. According to national guidelines [3, 4], patients at risk for HRMO are routinely screened at admission to reduce nosocomial spread. In addition, new policy pos- tulated hospital-wide active surveillance testing for MRSA, VRE and CPE in patients with prolonged hospitalization ( $\geq 14$ days) and subsequent isolation upon detection. No informed consent was required by the local medical ethics committee as the screening for HRMO at day 14 was part of the new policy and consid- ered as evaluation of care. We analysed data gathered between 15 December 2016 and 15 March 2018. Day 14 of hospitalization was chosen as an optimum in the bal- ance between the number of patients tested versus diag- nostic costs.}




\section{Specimen collection and microbiological procedures}

On day 14 of hospitalization an instruction appeared in the electronic patient record to collect a single set of swabs from nose, throat, and rectum for HRMOscreening. Screening for MRSA was performed with nose, throat and rectal swab samples, while VRE and $\mathrm{CPE}$ colonization was tested in rectal swabs only.

Detection of MRSA carriage was performed as described previously [7] and molecular typing of MRSAisolates by multiple locus variable number of tandem repeat analysis (MLVA) was performed at the RIVM, Bilthoven, The Netherlands. Detection of VRE was done by quantitative reverse-transcriptase polymerase chain reaction (RT-PCR) of $v a n A$ and $v a n B$ genes as described previously [8]. Molecular typing of VRE-positive isolates was performed at the University Medical Center Groningen, Groningen, The Netherlands. Carbapenemase resistance genes (bla $a_{\mathrm{KPC}}, b l a_{\mathrm{OXA}-48}, b l a_{\mathrm{NDM}}, b l a_{\mathrm{IMP}}$ and bla $\left.a_{\mathrm{VIM}}\right)$ were detected by RT-PCR as described previously [9].

Source and contact tracing investigations were performed by the infection-control team according to guidelines endorsed by the Dutch Working Party on Infection Prevention [4].

\section{Data collection and analysis}

A query was run in the General Laboratory Information Management System selecting patients hospitalized $\geq 14$ days, who underwent active surveillance for HRMO. Patient data including department, date of sample collection and microbiological result of active surveillance, were collected. Our primary outcome variable was the number of MRSA, VRE and CPE-carriers identified using active surveillance in patients hospitalized $\geq 14$ days. Secondary outcome variables included the number of newly identified MRSA-positive inpatients hospitalized $\geq 14$ days in relation to the total number of newly identified MRSA-positive inpatients, and the number of contact tracings indicating nosocomial transmission. Data were analysed using Excel (v14.7.2, Microsoft Corporation).

Costs of laboratory services for microbiological determinations were calculated.

\section{Results}

A total of 1899 screening sets from 1765 individual patients were collected and included in our analysis. The number of screening sets sampled each month gradually increased from 60 in the first month after policy change to a peak of 192 in the second last month (Fig. 1). Over the course of 15 months HRMO was detected in 24 previously unidentified patients $(1.36 \%$ [95\% confidence interval $\{C I\}, 0.92-2.02]$; Fig. 1).

MRSA colonization was discovered in 11 patients (0.62\% [95\% CI, 0.35-1.11]). Contact tracing investigations indicated that nosocomial transmission occurred twice. In the neonatology ward a nurse providing care for two twins who were found MRSA-positive in our screening programme (Fig. 1), was tested positive for MRSA as well as both parents. Other healthcare workers

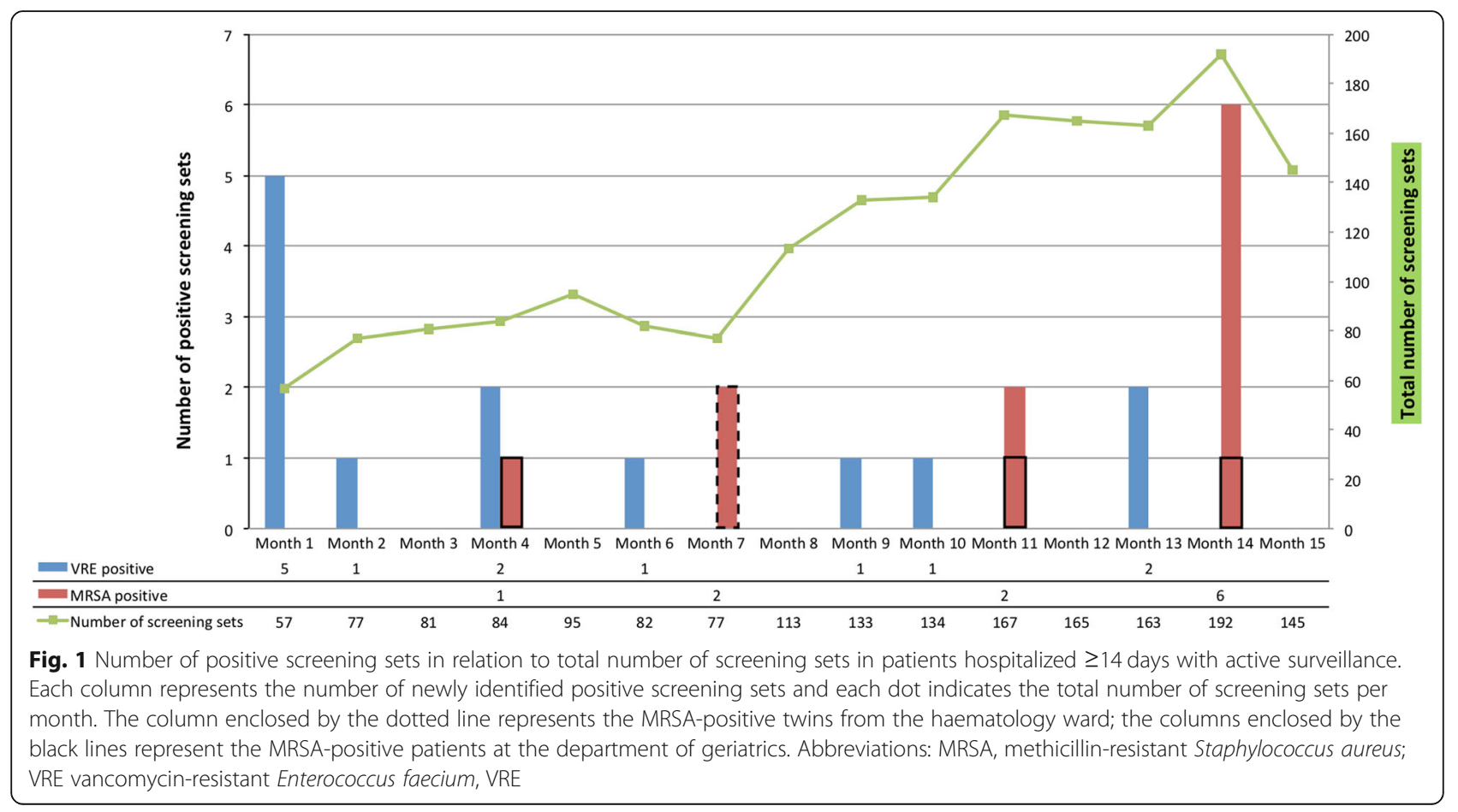




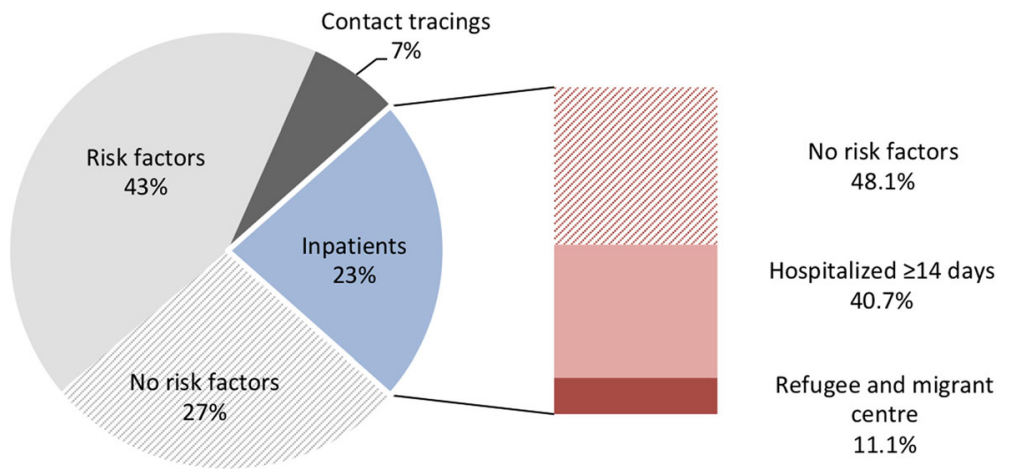

Fig. 2 Newly identified MRSA-positive patients. Each slice represents a percentage of a total of 117 MRSA carriers. The grey slices represent outpatients. Each block in the bar represents a percentage of a total of 27 MRSA-positive inpatients

screened were MRSA-negative $(n=85)$. Molecular typing confirmed nosocomial transmission showing a similar MLVA-profile in each of the isolates. At the department of geriatrics our surveillance strategy retrospectively identified a cluster of three MRSA-positive patients carrying an identical strain (Fig. 1). Notably, these patients had no overlapping period of hospitalization, suggesting presence of a common source although this was never proven by source and contact tracings including screening of patients and healthcare workers [3].

VRE was detected in 13 previously unidentified patients $(0.74 \%$ [95\% CI, 0.43-1.26]). A monoclonal outbreak of VRE was discovered before our study period and all VRE-isolates were vanA-positive belonging to the outbreak strain cgMLST 1026, as confirmed by whole genome sequencing. This is illustrated in Fig. 1 where the number of VRE positive patients is highest in the first month. No CPE-positive patients were detected.

During the study period a total of 117 patients were tested positive for MRSA in our laboratory (Fig. 2). Seventy-seven percent (90/117) of newly identified MRSA carriers were outpatients and 23\% (27/117) were hospitalized patients. Among the latter group, active screening of patients hospitalized $\geq 14$ days accounted for $40.7 \%(11 / 27)$ of newly identified MRSA carriers, while $48.1 \%(13 / 27)$ were unexpected results of clinical culture samples and $11.1 \%(3 / 27)$ were MRSA-positive patients from refugee and migrant centres (Fig. 2).

Costs of microbiological laboratory services for active screening of 1000 patients hospitalized $\geq 14$ days were calculated at $€ 120,000$, resulting in $€ 120$ per screened patient and $€ 227,880$ during our study period covering the processing of 1899 screening sets.

\section{Discussion}

Our study shows that active surveillance in patients hospitalized $\geq 14$ days can be used to identify asymptomatic HRMO colonization supporting early detection of nosocomial transmission. Although only $1.4 \%$ of screened patients tested positive for HRMO, our surveillance strategy revealed 2 new clusters of MRSA, disclosed $40.7 \%$ of newly identified inpatients colonized with MRSA, and detected 13 VRE-positive patients who turned out to be part of a recently recognized outbreak. Due to infection prevention and control measures including enforced hand hygiene and cleaning and disinfection, isolation precautions, clinical lessons to the staff, and microbiological screening of contact patients, further dissemination was prevented.

To prevent nosocomial transmission of HRMO, compliance with hygienic practices including transmissionbased precautions and environmental cleaning is of utmost importance. In medical settings with excessive workload where adherence to infection control precautions may be suboptimal, our surveillance programme will have most added value. It supports identification of HRMO carriers, thereby reducing the risk of nosocomial transmission and outbreaks, and it could be used as a proxy for infection control practices.

Our policy brings considerable costs, raising the question whether it is money well spent. Considering high costs of controlling a nosocomial outbreak [10], however, we believe that hospitals may benefit from extending active surveillance from traditional risk groups to patients with prolonged hospitalization as our policy contributes to timely control of HRMO spread. We assume that our program may be optimized by a differentiated approach in detection of HRMO species on selected wards at risk for dissemination of HRMO. Besides, screening of other patient categories that are potentially at risk for HRMO carriage such as patients who are transferred from chronic care facilities may contribute to control of HRMO in hospitals. More detailed, prospective studies are needed to address these issues.

\section{Abbreviations}

CPE: Carbapenemase-producing Enterobacteriales; HRMO: Highly resistant microorganisms; MLVA: Multiple locus variable number of tandem repeat analysis; MRSA: Methicillin-resistant Staphylococcus aureus; RT- 
PCR: Quantitative reverse-transcriptase polymerase chain reaction; VRE: Vancomycin-resistant Enterococcus faecium

\section{Acknowledgements}

We would like to thank Alma Tostmann for statistical support.

\section{Authors' contributions}

$\mathrm{CP}$ and EMM were responsible for the study design. GJHB and TB and EMM were responsible for data collection and analysis. GJHB and EMM drafted the manuscript while critical revision was provided by TB and CP. All authors read and approved the final version of the manuscript.

\section{Funding}

Not applicable.

\section{Availability of data and materials}

All data generated or analysed during this study are included in this published article.

\section{Ethics approval and consent to participate}

The study was judged to be beyond the scope of the Medical Research Involving Human Subjects Act (in Dutch, Wet Medischwetenschappelijk Onderzoek met Mensen [WMO]) by the ethics committee of the Rijnstate Hospital, and a waiver of written informed consent was obtained. The study was reviewed by the ethics committee on the basis of the Dutch Code of conduct for health research, the Dutch Code of conduct for responsible use, the Dutch Personal Data Protection Act and the Medical Treatment Agreement Act. The ethics committee has passed a positive judgment on the study.

\section{Consent for publication}

Not applicable.

\section{Competing interests}

The authors declare that they have no competing interests.

\section{Author details}

'Laboratory for Medical Microbiology and Medical Immunology, Rijnstate Hospital, Pres. Kennedylaan 100, 6883 AZ Velp, The Netherlands. ${ }^{2}$ Department of Medical Microbiology \& Radboud Center for Infectious Diseases, Radboud University Medical Center, Nijmegen, the Netherlands. ${ }^{3}$ Department of Infection Control, Rijnstate Hospital, Wagnerlaan 55, 6815 AD Arnhem, The Netherlands.

Received: 10 September 2019 Accepted: 23 December 2019 Published online: 07 January 2020

\section{References}

1. De Greef SC, Mouton JW, Schoffelen AF, Verduin CM. NethMap 2019: Consumption of antimicrobial agents and antimicrobial resistance among medically important bacteria in the Netherlands in 2018 [Internet]. 2019. Available from: https://www.rivm.nl/sites/default/files/2019-09/Nethmap Maran 2019 beveiligd.pdf.

2. Dutch Ministry of Health Welfare and Sport. Letter: voortgang aanpak antibioticaresistentie [Internet]. 2018. Available from: https://www. rijksoverheid.nl/documenten/kamerstukken/2018/04/26/kamerbrief-overvoortgang-aanpak-antibioticaresistentie

3. Dutch Working party on Infection Prevention. MRSA [Internet]. 2012. Available from: https://www.rivm.nl/sites/default/files/2018-11/121205 MRSA v1a def.pdf.

4. Dutch Working party on Infection Prevention. Measures to prevent the transmission of highly resistant microorganisms (HRMO) [Internet]. 2012. Available from: https://www.rivm.nl/sites/default/files/2018-11/130424 BRMO.pdf.

5. Buke C, Armand-Lefevre L, Lolom I, Guerinot W, Deblangy C, Ruimy R, et al. Epidemiology of multidrug-resistant bacteria in patients with long hospital stays. Infect Control Hosp Epidemiol. 2007;28:1255-60.

6. Friedmann R, Raveh D, Zartzer E, Rudensky B, Broide E, Attias D, et al. Prospective evaluation of colonization with extended-Spectrum $\beta$-lactamase (ESBL)-producing Enterobacteriaceae among patients at hospital admission and of subsequent colonization with ESBL-producing Enterobacteriaceae among patients during hospitalization. Infect Control Hosp Epidemiol. 2009; 30:534-42.

7. Nijhuis RHT, van Maarseveen NM, van Hannen EJ, van Zwet AA, Mascini EM. A rapid and high-throughput screening approach for methicillin-resistant Staphylococcus aureus based on the combination of two different real-time PCR assays. J Clin Microbiol. 2014;52:2861-7.

8. Sinnige JC, Willems RJL, Ruijs GJHM, Mascini E, Arends JP, Troelstra A. NVMM Guideline HRMO VRE; 2015.

9. Nijhuis R, Samuelsen $\varnothing$, Savelkoul P, van Zwet A. Evaluation of a new realtime PCR assay (check-direct CPE) for rapid detection of KPC, OXA-48, VIM, and NDM carbapenemases using spiked rectal swabs. Diagn Microbiol Infect Dis. 2013;77:316-20.

10. Dik JWH, Dinkelacker AG, Vemer P, Lo-Ten-Foe JR, Lokate M, Sinha B, et al. Cost-analysis of seven nosocomial outbreaks in an academic hospital. PLoS One. 2016;11(2):e0149226.

\section{Publisher's Note}

Springer Nature remains neutral with regard to jurisdictional claims in published maps and institutional affiliations.

\section{Ready to submit your research? Choose BMC and benefit from:}

- fast, convenient online submission

- thorough peer review by experienced researchers in your field

- rapid publication on acceptance

- support for research data, including large and complex data types

- gold Open Access which fosters wider collaboration and increased citations

- maximum visibility for your research: over $100 \mathrm{M}$ website views per year

At BMC, research is always in progress.

Learn more biomedcentral.com/submissions 